Review

\title{
Perioperative Contamination of Orthopaedic Polyethylene Implants, Targeting Devices and Arthroscopes. Experts' Decision Tree and Literature Review
}

\author{
Matthias Vautrin ${ }^{凶}$, Kevin Moerenhout, Gilles Udin, Olivier Borens \\ Orthopaedic Trauma Surgery Unit, Department of the Locomotor Apparatus, Lausanne. University Hospital, Rue du Bugnon 46, CH-1011, Lausanne, \\ Switzerland. \\ $\triangle$ Corresponding author: Dr Matthias Vautrin, Centre Hospitalier Universitaire Vaudois, Rue du Bugnon 46, CH-1011 Lausanne, Switzerland. Email: \\ matthias.vautrin@chuv.ch \\ (C) Ivyspring International Publisher. This is an open access article distributed under the terms of the Creative Commons Attribution (CC BY-NC) license \\ (https://creativecommons.org/licenses/by-nc/4.0/). See http://ivyspring.com/terms for full terms and conditions.
}

Received: 2018.10.12; Accepted: 2019.01.26; Published: 2019.02.16

\begin{abstract}
Introduction Sterility errors during orthopaedic procedures can be stressful for the surgeon or scrub nurse and lead to devastating infectious complications and liability issues. This paper aims to review orthopaedic surgeon practices and propose possible attitudes to adopt.

Methods Out of 1023 questionnaires sent, 170 orthopaedic surgeons answered a Volunteer Feedback Template (multiple-choice test) by SurveyMonkey ${ }^{\circledR}$ (San Mateo, CA, USA) anonymously. The survey questioned surgeon's response to a sterility mistake during a standard total knee joint replacement, trauma surgery and arthroscopic procedure. Those "sterility mistake" situations occurred when there was contamination of 1) a sterile polyethylene (PE) 2) a sterile targeting device, and 3) an arthroscope.

Results When the definitive PE is contaminated, and if a new definitive PE will only be available 2 hours later, $52 \%$ of surgeons would wait for the new definitive PE $(p<0.001)$. In the same situation, if a new PE will only be available in 4 hours, the results showed a significant difference favoring two other options: "putting a definitive PE one size smaller or bigger with balance adjustment" (31\%); and "leaving the provisional PE in the joint, closing the wound and re-operating the patient in the coming days when the definitive PE arrives" $(29 \%)(p<0.001)$. When the new PE is only available 24 hours later results were $34 \%$ and $31 \%$, respectively $(p<0.001)$. In the case of a surgical procedure for a classic intertrochanteric fracture, if the carbon fiber targeting device is contaminated, most surgeons (50\%) chose to put the nail without the targeting device and finish the surgery $(\mathrm{p}<0.001)$. When the arthroscope is desterilized, $39 \%$ of participants would wait until the arthroscope has been sterilized again (approximately 2 hours), while $24 \%$ would use another procedure $(p<0.001)$. Sixty-two percent of surgeons would adapt their strategy. No clear trend could be identified in terms of antibiotic treatment following a sterility error.

Conclusions There are no established guidelines on how to deal with sterility breaches during surgery and on the antibiotic strategy following the prolonged surgical time resulting from the delay for a new implant. The most common course of action chosen by participating surgeons is detailed in our expert decision tree - if another sterile component is not available within 2 hours - : insertion of another PE size, rescheduling the operation, adapting the surgical technique (for trauma procedures), or soaking the arthroscope in disinfectant solution. As instances of contamination cannot be avoided, it is recommended to have a minimum of two copies of sterile PE implants, arthroscopes or targeting devices readily available before surgery begins-.
\end{abstract}

Key words: perioperative contamination, polyethylene implants, targeting devices, arthroscopes, fall to the ground, unsterile surface, orthopaedic surgery, expert opinion 


\section{Introduction}

There are many challenges associated with surgery and even common orthopaedic procedures, such as a total hip or total knee arthroplasty (THA-TKA), can be complicated by sterility issues. Indeed, a trial implant, or worse a definitive implant, falling to the ground, or a surgical scrub failure can impact the final outcome. Addressing such events should be based on the literature or previous research as much as possible. If a replacement for the unsterile implant is directly at hand, decision-making is easy but there are no clear guidelines when that is not the case. The literature is also very sparse on what to do when a sterility error occurs during surgery, although an unsterilized orthopaedic implant with bacterial contamination can lead to a disastrous outcome [1].

Due to the lack of clear guidelines, and in order to define the best possible strategy, our primary aim was to determine how orthopaedic surgeons would handle such sterility issues. Our secondary goal was to evaluate if an experienced surgeon would act differently from an inexperienced surgeon, taking into account the available material and work environment.

\section{Methods}

\section{Literature search}

A systematic search of three bibliographic databases (Embase.com, PubMed and Cochrane Central) found no definite guidelines with the following keywords: intra-operative bacterial contamination of implants, falling implants, polyethylene liners, instruments, targeting device, arthroscope, fall to ground, unsterile surface, bacterial contamination, bacteria, germs, microbes, microorganism, air contamination, skin contamination, skin preparation, unsterile surface, draping defects, glove defects, orthopaedic operation, expert opinion, recommendation, prosthesis infection PI, joint prosthesis infection, prosthesis joint infection PJI, infected arthroplasty, prosthesis infection, decontamination procedure, antiseptic solution, antimicrobial agents, wound antiseptic, antiseptic, antimicrobial agents, bactericidal activity, prevention of surgical side infection (SSI), antiseptic solution, irrigation, antiseptics, orthopaedic surgery, sterility, expert opinion.

\section{Questionnaire development}

The authors, orthopaedic trauma surgeons and specialists in septic surgery, developed a questionnaire based on established survey design guidelines [2].

\section{Questionnaire distribution}

A Volunteer Feedback Template (multiple choice test) by SurveyMonkey ${ }^{\circledR}$ (San Mateo, CA, USA) was sent to the residents and orthopaedic specialists of the Lausanne Orthopaedic Trauma Surgery Unit $(n=36)$, to the members of Swiss Orthopaedics $(n=580)$ and the members of the European Bone and Joint Infection Society $(n=407)$. A total of 1023 questionnaires were sent. Surgeons were asked to answer 10 questions describing "typical" orthopaedic, traumatology or sports medicine situations involving sterility mistakes. The questionnaire, which remained strictly anonymous, was sent on the $2^{\text {nd }}$ of March 2017 and was closed on the $30^{\text {th }}$ of March 2017. All the data were extracted (independently) by the main author according to the Volunteer Feedback Template. Similarly, Morgenstern et al. [3] conducted a survey on the definition of fracture related infections in which a questionnaire was sent to all registered $\mathrm{AO}$ Trauma users (Davos, Switzerland).

\section{Population}

A total of 170 surgeons and residents answered the multiple-choice test. Forty-four percent of surgeons were affiliated to a university hospital, $32 \%$ to a periphery hospital, $21 \%$ to a private clinic, and $2 \%$ elsewhere.

Orthopaedic subspecialties were divided between arthroplasty (45\%), traumatology (18\%), septic surgery $(14 \%)$, sports medicine $(6 \%)$, and other $(18 \%)$. Among 170 participants, $14 \%$ had been awarded their orthopaedic specialist certificate less than 5 years previously, 21\% had been certified between 5 and 10 years, $53 \%$ for more than 10 years, and $12 \%$ were still in training.

\section{Statistical analysis}

Statistical analysis was performed with Jamovi 0.8.6. The primary analysis focused on answers that would be over or under represented, compared to a random distribution. We then analyzed the answers according to participants' subspecialty (traumatology, arthroplasty, septic surgery, or sports medicine). When the results from one subspecialty significantly differed from the rest, each possible response mode (for example answer mode 1 to 6 for PE) was evaluated to determine where the difference occurred. Finally, we tested whether results differed between participants coming from a University hospital, a periphery hospital or a private clinic. Unless stated otherwise, statistical significance was calculated as: $\chi^{2}$ x (5 df, $\mathrm{N}=170), \mathrm{p}<0.05$.

\section{Results}

Over the course of their practice, nearly half of the participants $(47 \%)$ reported having modified their surgical strategy between one and five times, $15 \%$ more than five times and 38\% never had to adapt their 
strategy because of a sterility error.

\section{Sterile PE falls to the floor}

For this question, we began by analyzing all the responses, and then compared the answers of the experienced surgeons (certified for more than 10 years, affiliated to the university, specialized in traumatology and septic surgery) to those of inexperienced surgeons (certified less than 5 years, peripheral and private hospitals, not specialized in traumatology and septic surgery).

During a total knee arthroplasty (TKA) procedure, the definitive polyethylene (PE) falls on the ground. What would you do if a new definitive PE is available only in 2 hours?

\section{Global opinion}

In this type of situation, $52 \%$ of surgeons would wait until the new definitive PE arrives $(p<0.001)$; the other options were less popular. (Figure 1)

\section{Experienced vs inexperienced}

Fifty-six percent of experienced surgeons would wait until the new definitive PE arrives $(p<0.05)$. Other solutions, such as soaking the fallen PE implant (23\%), adapting the PE size (14\%), or putting the provisional $\mathrm{PE}$ in the joint $(9 \%)$, were chosen less frequently $(\mathrm{p}<0.05)$.

Among the inexperienced surgeons, 50\% would wait for the new definitive PE , 33\% would leave the provisional PE in the joint, close the wound, and re-operate the patient in the coming days when the definitive PE arrives, and $17 \%$ did not have an answer $(\mathrm{p}<0.05)$.

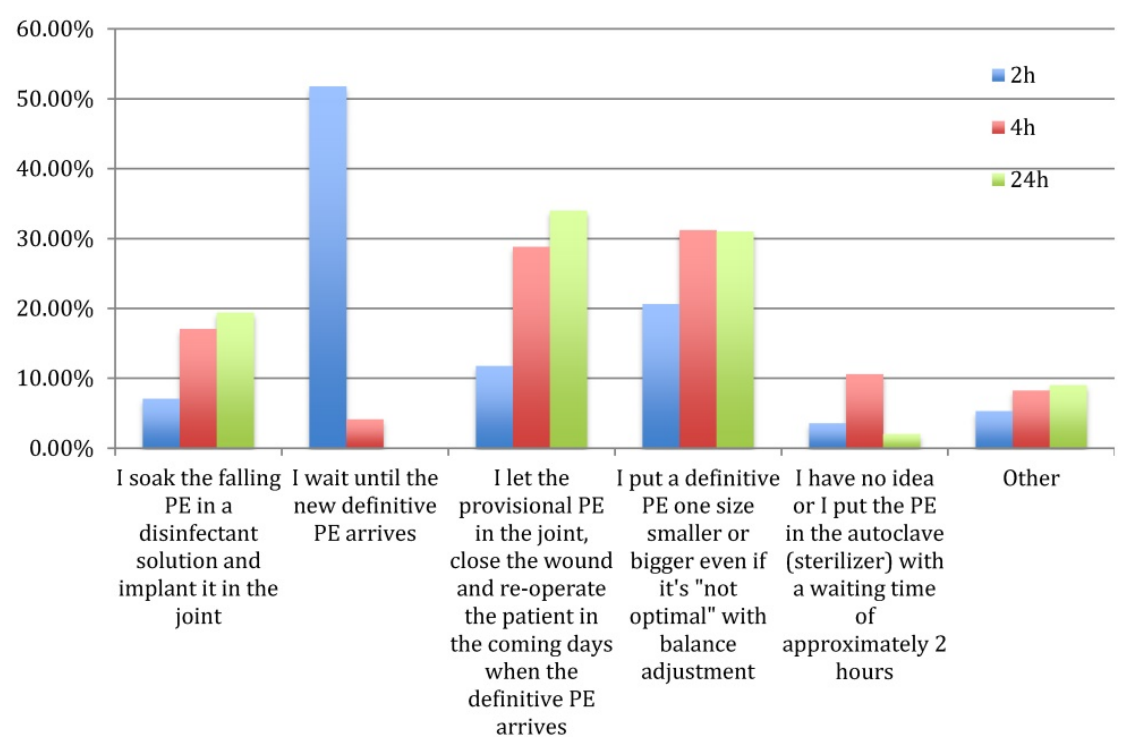

Figure 1. Comparison between three possible situations. 'During a total knee arthroplasty (TKA) procedure, the definitive polyethylene (PE) falls on the ground. What would you do if a new definitive PE is available only in 2,4 or 24 hours?'
During a total knee arthroplasty (TKA) procedure, the definitive polyethylene (PE) falls on the ground. What would you do if a new definitive PE is available only in 4 hours?

\section{Global opinion}

In this situation, most participants selected between two options: "putting a definitive PE one size smaller or bigger with balance adjustment" (31\%); and "leaving the provisional PE in the joint, closing the wound and re-operating the patient in the coming days when the definitive PE arrives" $(29 \%)(\mathrm{p}<0.001)$. (Figure 1)

\section{Experienced vs inexperienced}

Most experienced surgeons would "put a definitive PE one size smaller or bigger even if it's "not optimal" with balance adjustment" (41\%) and 27\% would prefer "soaking the implant in disinfectant"; $18 \%$ would "leave the provisional PE in the joint, close the wound, and re-operate the patient in the coming days when the definitive PE arrives".

Among the inexperienced surgeons, 50\% would "leave the provisional PE in the joint, close the wound, and re-operate the patient in the coming days when the definitive PE arrives"; 17\% would "put a definitive PE one size smaller or bigger even if it's not optimal with balance adjustment"; $17 \%$ would prefer "soaking the implant in disinfectant" and 17\% would choose another solution $(\mathrm{p}<0.05)$.

During a total knee arthroplasty (TKA) procedure, the definitive polyethylene (PE) falls on the ground. What would you do if a new definitive PE is available only in

\section{4 hours?}

\section{Global opinion}

Two options were chosen significantly more often: "putting a definitive PE one size smaller or bigger with balance adjustment" (34\%); and "leaving the provisional $\mathrm{PE}$ in the joint, closing the wound, and re-operating the patient in the coming days when the definitive PE arrives" $(31 \%)(p<0.001)$. (Figure 1$)$

\section{Experienced vs inexperienced}

Experienced surgeons prefer "soaking the implant in disinfectant" $(32 \%)$ or "putting a definitive PE with adapted balance adjustment $(32 \%)^{\prime \prime}$.

Inexperienced surgeons would leave the provisional PE in the joint, close the wound, and re-operate the 
patient in the coming days when the definitive PE arrives (67\%), 17\% would soak the implant in disinfectant and $17 \%$ would find another solution $(\mathrm{p}<0.05)$.

\section{Sterile targeting device falls to the floor}

You are operating a pertrochanteric fracture in a periphery hospital. You are reaming your femur. Unfortunately, the carbon fiber targeting device falls on the ground. Another targeting device will only be available in 3 hours. What would you do?

For this question, we began by analyzing all the responses and then compared the answers of the experienced surgeons (certified for more than 10 years, affiliated to the university, specialized in traumatology and septic surgery) to those of inexperienced surgeons (certified for less than 5 years, peripheral and private hospitals, not specialized in traumatology and septic surgery).

\section{Global opinion}

In the case of a classic pertrochanteric fracture, if the carbon fiber targeting device falls to the ground, most surgeons (50\%) would choose to "put the nail without the targeting device and finish the surgery" $(\mathrm{p}<0.001)$. (Figure 2)

\section{Experienced vs inexperienced}

Experienced surgeons would "try to put the nail without the targeting device and finish the surgery" $(40 \%)$. Thirty-three percent would "soak the targeting device in disinfectant", 13\% would "use another type of implant even if it's not ideal".

Most inexperienced surgeons would try to "put the nail without the targeting device and finish the surgery" (67\%); 17\% would "soak it in disinfectant" and $16 \%$ did not have an answer.

\section{Contamination of an arthroscope}

You are planning to do meniscal suture during arthroscopy. During your installation, you desterilize your arthroscope. No other arthroscope is available. What would you do?

For this question, we began by analyzing all the responses and then compared the answers of the experienced surgeons (certified for more than 10 years, affiliated to the university, specialized in traumatology and septic surgery) to those of inexperienced surgeons (certified for less than 5 years, peripheral and private hospital, not specialized in traumatology and septic surgery).

\section{Global opinion}

When the arthroscope is desterilized, we found that $39 \%$ of participants would wait until the arthroscope is sterile once again (approximately 2 hours), while $24 \%$ would use another procedure $(\mathrm{p}<0.001)$. (Figure 3)

\section{Experienced surgeons vs inexperienced surgeons}

Thirty-one percent of experienced surgeons would soak the arthroscope in disinfectant; 31\% would wait until the arthroscope has been sterilized once again; and $16 \%$ would convert to arthrotomy.

Among inexperienced surgeons, 62\% would wait until the arthroscope has been sterilized once again, 25\% would soak it in disinfectant, and 13\% would choose another strategy $(\mathrm{p}<0.05)$.

\section{Change of antibiotic regime after intra-operative contamination}

You make a sterility mistake during surgery. How long would you put the patient under antibiotics?

For this question, we began by analyzing all the responses and then compared the answers of the experienced surgeons (certified for more than 10 years, affiliated to the university, specialized in traumatology and septic surgery) to those of inexperienced surgeons (certified less than 5 years, peripheral and private hospitals, not specialized in traumatology and septic surgery).

\section{Global opinion}

A majority of surgeon would favour 24 hours of antibiotics $(p=0.0039)$ (Figure 4$)$

Experienced surgeons vs inexperienced surgeons

Results were similar for experienced surgeons and inexperienced surgeons ( $p=0.27)$; in all cases " 24 hours of antibiotics" was the most popular option.

\section{Discussion}

When a PE sterility mistake occurs during a TKA and the waiting time is under 2 hours most surgeons, whether experienced or inexperienced, would wait until a new PE arrives. This suggests that a two hour increase in surgical duration would be an acceptable threshold for most surgeons, regardless of any other alternative. The second most popular option was soaking the PE in disinfectant for experienced surgeons, while inexperienced surgeons would leave the provisional PE in the joint and re-operate the patient a few days later.

When the delay exceeds 2 hours none of the surgeons would choose to wait until the new PE arrives. This might be due to the greater risk of infection associated with increased surgical duration [4]. Most experienced surgeons would choose to put a definitive PE one size smaller or to soak the PE in a disinfectant solution, while most inexperienced 
surgeons prefer leaving the provisional PE in the joint, closing the wound and re-operating the patient in the coming days when the definitive PE arrives. Arthroplasty surgeons favored the first option. This might be explained by their comfort level, when it comes to adjusting the prosthesis, compared to other subspecialty participants. None of the inexperienced surgeons would choose to adapt the PE size. Again, this could be the result of a lack of familiarity with resizing a prosthetic implant.

When it came to a sterility mistake during a pertrochanteric procedure, most surgeons answered that they would insert the nail without the targeting device and finish the surgery. Since there are many available treatment options, with different implants or techniques, surgeons might feel more confident and therefore more willing to adapt their surgical technique. None of the surgeons, whether experienced or inexperienced, would wait for a new device. One third of experienced surgeons would still soak the device in disinfectant solution.

The question on sports medicine showed that most participants would wait 2 hours for the new arthroscope to arrive, while performing an arthrotomy was the second most popular answer. Experienced surgeons were almost equally divided between soaking the arthroscope in disinfectant solution and waiting for the newly sterilized arthroscope. Balato et al. published a review on thirteen studies where the incidence of infection ranged between 0.009 and $1.1 \%$ in patients undergoing simple arthroscopic procedures. Staphylococci are the most commonly isolated organisms in infections after arthroscopy [5]. Septic arthritis of the knee after arthroscopy causes significant morbidity, usually requiring readmission to the hospital, at least one additional operation, and prolonged antibiotic therapy.

The results to the question on the duration of the antibiotics treatment following a sterility mistake showed that "24-hour $\mathrm{AB}^{\prime \prime}$ was the most represented. Experienced surgeons never chose to put a single dose of antibiotics and most recommended a 24-hour antibiotics treatment duration. The answers for inexperienced surgeons were randomly distributed across all propositions.

The use of prophylactic antibiotics in orthopaedic surgery can effectively reduce SSI rates, as shown in studies on hip and knee arthroplasty, spine surgery, and open reduction and internal fixation of fractures. To maximize the beneficial effects of prophylactic antibiotics, while minimizing the adverse effects, the correct antimicrobial must be selected, the drug must be administered just before incision, and duration should not exceed 24 hours [6].

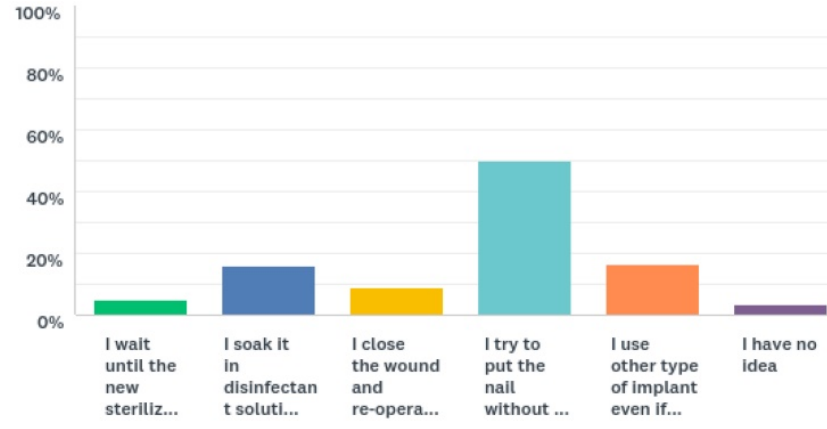

Figure 2. In the event of a sterility mistake during a trauma procedure.

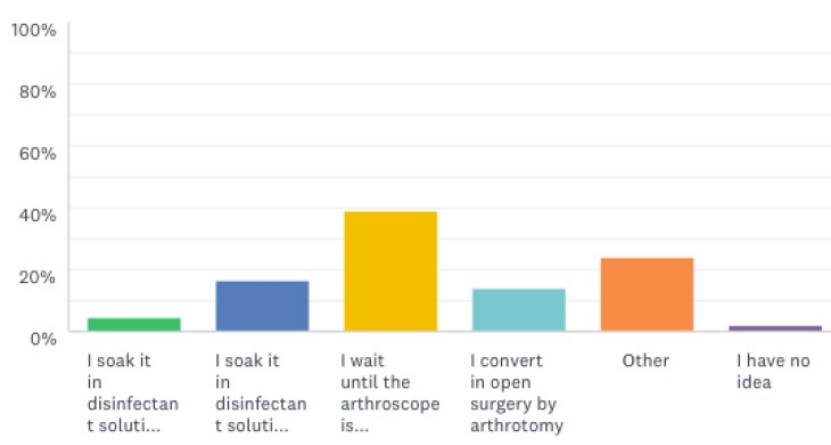

Figure 3. In the event of a sterility mistake during a sports medicine procedure.

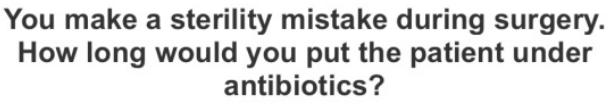
antibiotics?

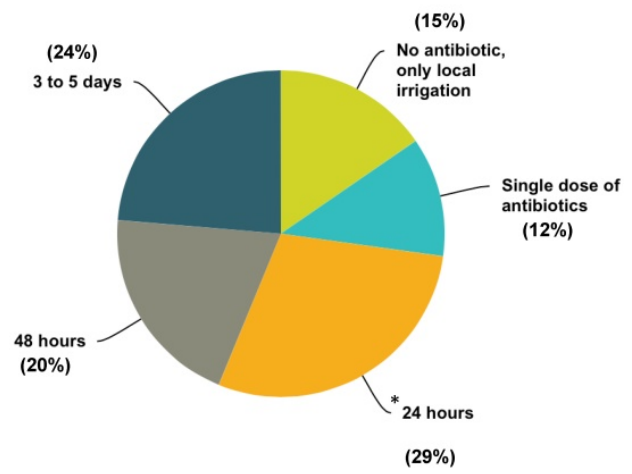

Figure 4. Global opinion on antibiotics treatment duration.

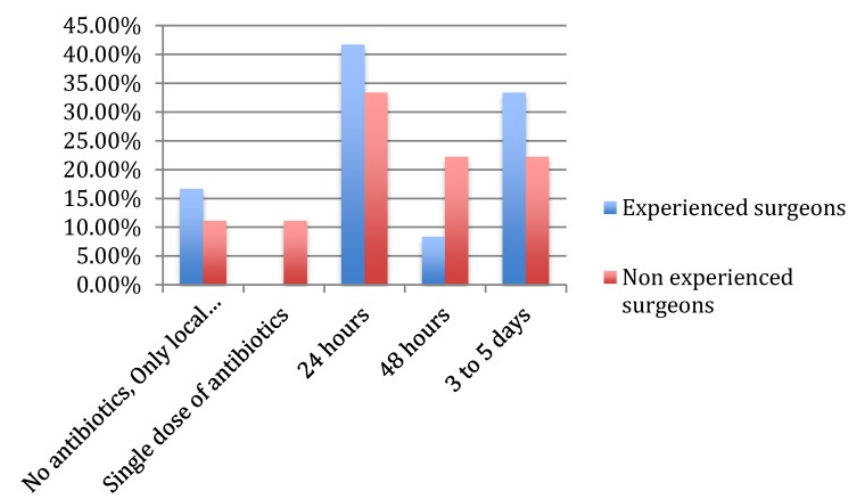

Figure 5. Comparison between experienced vs inexperienced surgeons for $A B$ duration. 
The benefits of antibiotic prophylaxis have been clearly established in standard procedures, with recommendations for timing and dosage [7]. However, there are no guidelines in cases of unexpected sterility errors. Treating infection at the surgical site is a constant challenge in orthopedic surgery, and remains a leading cause of failure, with increased morbidity and mortality rates [8]. Despite continued efforts in research, education and technical progress, per-operative contamination remains relatively frequent. An in-depth look at elective prosthetic surgery found up to $63 \%$ contamination of the surgical field (including sucker tips, light handles, skin blades or gloves, [9]). These per-operative contamination sites can originate from many sources such as: air [10], gloves or gown [11], or instrument trays [12]. Whyte, in 1982 [13], estimated that the contamination source was the surgeon or an instrument in approximately $70 \%$ of cases. Further studies showed that surgeons with a low volume of surgeries tend to have higher infection rates [14] (potentially resulting from increased surgical duration). Another study found no difference in hip fracture surgery outcome between a specialist hip unit and a general orthopedic surgeon [15].

In the sterility mistake situations described here two effects could be involved. First, the use of a contaminated implant or instrument clearly creates an additional potential source of surgical site infection.
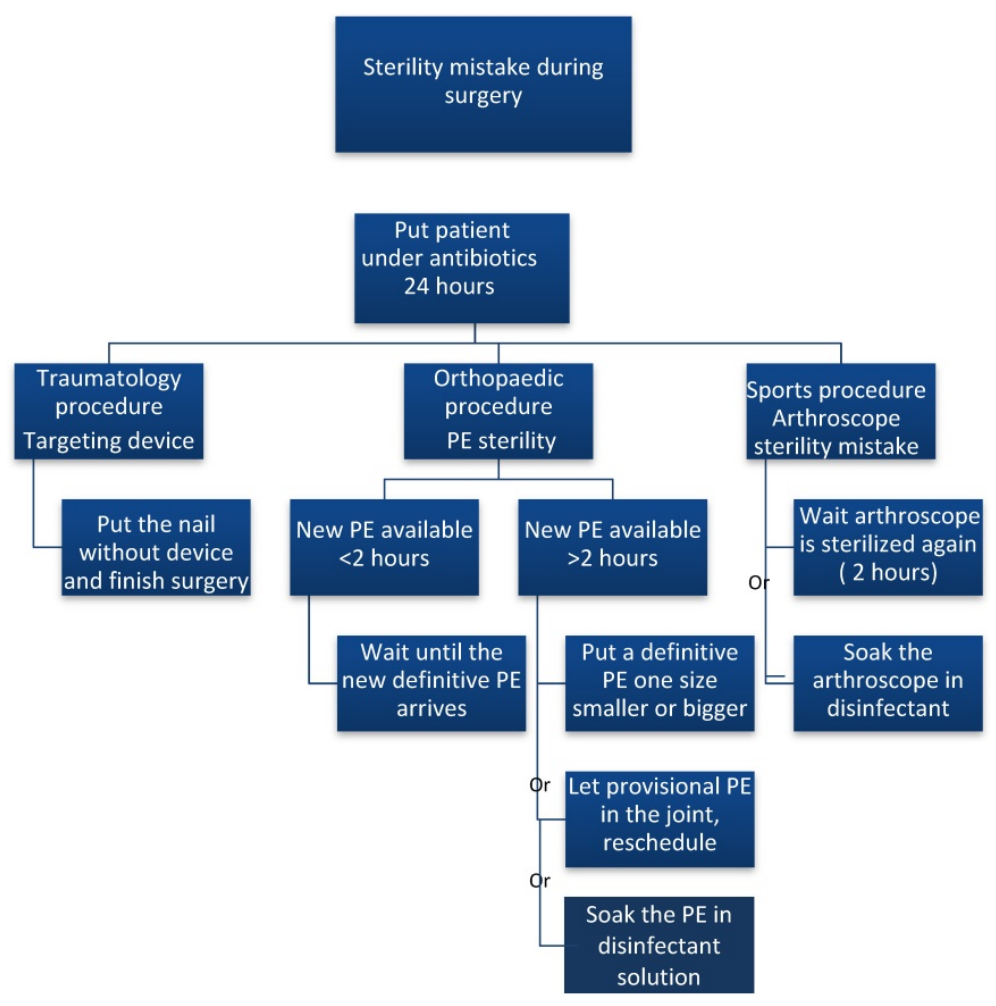

Figure 6. Decision tree based on experienced surgeons' opinion.
Due to obvious methodological limitations one can only extrapolate from non-dedicated studies. Ruder et al. [8] found that lavage of the contaminated implant reduced the risk of deep surgical site infection in orthopedic surgery, when using dilute povidoneiodine lavage prior to wound closure. As this chemical has no associated adverse effects, they concluded that this method is a safe and inexpensive method to reduce the infection rate. However, a clear regimen or procedure cannot be recommended based on the current literature [16]. Secondly, an instrument or an implant falling to the ground will increase surgical duration. An average delay of 7.6 minutes [17] consecutive to such an incident has been reported. This is an added risk factor, as a prolonged operating time is a recognized infection risk factor for hip and knee arthroplasty [18]. Indeed, procedures lasting more than 120 minutes have been associated with higher infection risks. Thus, regardless of implant contamination, the accident itself increases the risk of infection. Furthermore, the decision to wait two hours or more for a new implant will add to this risk. Current data does not provide a clear picture of the relative risks involved in either using a contaminated implant, increasing surgical duration, or performing a second surgery. Each option increases the infection risk, but, to the best of our knowledge, no recommendations can be made on an evidence-based rationale.

Although many surgeons have been exposed to the above-mentioned unpleasant situations, not enough has been done to create a working group and establish consensus on the best course of action when it occurs. In the meantime, based on the answers given by the participants, we created a decision tree that reflects what the most experienced participants would do. (Figure 6)

One of the most important limitations of our study consists in the low number of participants. However, the respondents surveyed are a representative sample of the orthopaedic surgeon population. The strength of our article is that, to the best of our knowledge, it is the first study to propose a decision tree in a sterility mistake situation.

An implant accidentally falling to the floor is one of the hazards of orthopaedic surgery. The present paper reported how experienced and inexperienced surgeons would handle the intra-operative contamination of sterile implants or devices. Based on this data we created a decision tree detailing the course of action that the most 
experienced surgeons would follow in such a situation. Finally, we strongly recommend having a minimum of two copies of every prosthetic implant size or orthopaedic device as backup when starting surgery.

\section{Abbreviations}

PE: definitive polyethylene; THA: Total hip arthroplasty; TKA: Total knee arthroplasty; FRI: Fracture related infection; AB: antibiotic; SSI: Surgical Site Infections.

\section{Acknowledgements}

MV collected and interpreted the data, wrote the manuscript and acted as corresponding author. KM designed the study, supervised development of work, helped in data interpretation and manuscript interpretation. GU performed the analysis. OB developed the methodology, helped to evaluate and edit the manuscript.

\section{Competing Interests}

The authors have declared that no competing interest exists.

\section{References}

1. Ribeiro M, Monteiro FJ, Ferraz MP. Infection of orthopedic implants with emphasis on bacterial adhesion process and techniques used in studying bacterial-material interactions. Biomatter. 2012 Oct 1; 2(4): 176-194.

2. Burns KE, Duffett M, Kho ME, et al. A guide for the design and conduct of self-administered surveys of clinicians. CMAJ. 2008; 179(3): 245-52.

3. Morgenstern $\mathrm{M}$, Moriarty $\mathrm{TF}$, Kuehl $\mathrm{R}$ et al. International survey among orthopaedic trauma surgeons: Lack of a definition of fracture-related infection Injury. 2018; Feb 6. pii: S0020-1383(18)30058-5

4. Cheng H, Chen BP, Soleas IM, et al. Prolonged Operative Duration Increases Risk of Surgical Site Infections: A Systematic Review. Surg Infect (Larchmt). 2017 Aug 1; 18(6): 722-735.

5. Balato G, Di Donato SL, Ascione $\mathrm{T}$ et al. Knee Septic Arthritis after Arthroscopy: Incidence, Risk Factors, Functional Outcome, and Infection Eradication Rate. Joints. 2017; 5(2): 107-113.

6. Prokuski, L, Clyburn TA, Evans RP, et al. Prophylactic antibiotics in orthopaedic surgery. Instr Course Lect. 2011; 60: 545-55

7. van Kasteren ME, Manniën J, Ott A, et al. (2007). Antibiotic Prophylaxis and the Risk of Surgical Site Infections following Total Hip Arthroplasty: Timely Administration Is the Most Important Factor. Clin Infect Dis. $2007 \mathrm{Apr}$ 1;44(7):921-7.

8. Ruder JA, Springer BD. Treatment of Periprosthetic Joint Infection Using Antimicrobials: Dilute Povidone-Iodine Lavage. J Bone Jt Infect. 2017 Jan 1;2(1):10-14.

9. Knobben BA, Engelsma Y, Neut D, et al. Intraoperative contamination influences wound discharge and periprosthetic infection. Clin Orthop Relat Res. 2006 Nov; 452:236-41.

10. Parvizi J, Barnes S, Shohat N, Edmiston CE Jr. Environment of care: Is it time to reassess microbial contamination of the operating room air as a risk factor for surgical site infection in total joint arthroplasty? Am J Infect Control. 2017 Nov 1:45(11):1267-1272.

11. Ward WG Sr, Cooper JM, Lippert D, et al. Glove and gown effects on intraoperative bacterial contamination. Ann Surg. 2014 Mar;259(3):591-7.

12. Bible JE, O'Neill KR, Crosby CG, et al. Implant contamination during spine surgery. Spine J. 2013 Jun;13(6):637-40.

13. Whyte W, Hodgson R, Tinkler J. The importance of airborne bacterial contamination of wounds. J Hosp Infect. 1982 Jun;3(2):123-35.

14. Muilwijk J, van den Hof S, Wille JC. Associations between surgical site infection risk and hospital operation volume and surgeon operation volume among hospitals in the Dutch nosocomial infection surveillance network. Infect Control Hosp Epidemiol. 2007 May; 28(5):557-63.

15. Guerado E, Cano JR, Cruz E, et al. Should hip fractures be operated upon only by specialist hip unit surgeons in order to lower rates of surgical site infection? Inter Orthop. 2015; 39(1): 105-110.
16. Lüdemann M, Munoz P, Wagner $\mathrm{M}$, et al. The Effect of Antiseptics in the Prophylaxis of Infection in Orthopaedic Surgery. Z Orthop Unfall. 2018 Oct;156(5):567-573.

17. Khan SA, Kumar A, Varshney MK et al. Accidentally falling instruments during orthopaedic surgery: time to wake up! ANZ J Surg. 2008; 78(9): 794-5

18. Willis-Owen CA, Konyves A, Martin DK. Factors affecting the incidence of infection in hip and knee replacement: an analysis of 5277 cases. J Bone Joint Surg Br. 2010 Aug; 92(8): 1128-33. 